\title{
A-Optimal versus D-Optimal Design of Screening Experiments
}

\author{
Bradley Jones \\ SAS Institute \\ Katherine Allen-Moyer \\ Department of Statistics \\ North Carolina State University \\ Peter Goos \\ Faculty of Bioscience Engineering and Leuven Statistics Research Centre \\ KU Leuven
}

August 24, 2020

\begin{abstract}
The purpose of this article is to persuade experimenters to choose A-optimal designs rather than D-optimal designs for screening experiments. The primary reason for this advice is that the A-optimality criterion is more consistent with the screening objective than the D-optimality criterion. The goal of screening experiments is to identify an active subset of the factors. An A-optimal design minimizes the average variance of the parameter estimates, which is directly related that goal. While there are many cases where A- and D-optimal designs coincide, the A-optimal designs tend to have better statistical properties when the A- and D-optimal designs differ. In such cases, A-optimal designs generally have more uncorrelated columns in their model matrices than D-optimal designs. Also, even though A-optimal designs minimize the average variance of the parameter estimates, various cases exist where they outperform D-optimal designs in terms of the variances of all individual parameter estimates. Finally, A-optimal designs can also substantially reduce the worst prediction variance compared to D-optimal designs.
\end{abstract}

Keywords: main effect, orthogonal array, prediction variance, two-factor interaction effect, two-level design. 


\section{Introduction}

In recent years, optimal experimental designs have replaced standard designs from catalogs in many applications. Multiple optimality criteria exist for selecting the optimal experimental designs. The D- and A-optimality criteria focus on precise estimation of the model parameters and are therefore estimation-oriented. The I- and G-optimality criteria focus on precise predictions of the response and are therefore prediction-oriented. In this article, we focus on screening experiments and compare the D- and A-optimality criteria. This is because the main goal of screening is to detect active effects. This argues for precise parameter estimates yielding higher powers for detecting active effects. The estimation-oriented D- and A-optimality criteria are therefore more suitable to design screening experiments than the prediction-oriented I- and G-optimality criteria. Our work is inspired by the fact that the A-optimality criterion is easier to explain than the D-optimality criterion. The A-optimality criterion focuses on the variances of the parameter estimates (whose square roots are used by every practitioner who performs significance tests and build confidence intervals in factor screening), while the D-optimality criterion minimizes the volume of a confidence ellipsoid about the parameters (which has little practical relevance to a practitioner).

The first algorithms for creating designed experiments using a computer employed the D-optimality criterion and produced highly D-efficient designs (see, for instance, Mitchell (1974), Cook and Nachtsheim (1980, 1989) and Atkinson and Donev (1989)). When the goal is factor screening, the desired model is the main-effects model, and the number of runs is a multiple of four, the D-optimal design is an orthogonal array. Many optimal design algorithms are able to construct orthogonal arrays for these cases when the number of runs and the number of factors are not overly large. This fact bolsters the credibility of computer-generated D-optimal screening designs even when the desired number of runs is not a multiple of four and the desired model includes the main effects and the two-factor interactions. Therefore, D-optimal designs have often been recommended for performing screening experiments (see, for instance, Goos and Jones (2011)).

Algorithms and software for generating A-optimal designs are scarce compared to algorithms and software for generating D-optimal designs. Likewise, there is far more literature 
on D-optimal designs than on A-optimal designs. One reason for this is that computer searches for A-optimal designs require the computation of the inverse of the information matrix, while searches for D-optimal designs only require the determinant of the information matrix to be computed. The latter is computationally less expensive than the former, as a result of which computing D-optimal designs requires less time than computing A-optimal designs. Another reason why A-optimal designs are less commonly used than D-optimal designs is that, unlike D-optimal designs, A-optimal designs are not invariant to changes in the scale or the coding of the factors (Atkinson and Donev; 1992; Goos; 2002). A third reason for the relative lack of popularity of A-optimal designs is that D-optimal designs are often said to perform well with respect to other optimality criteria (Atkinson and Donev; 1992; Goos; 2002). Finally, in many cases where the optimal designs are known, D-optimal designs are also A-optimal, and vice versa. For instance, for main-effects models involving two-level categorical factors or quantitative factors, orthogonal arrays are both D- and A-optimal.

In this article, we compare A-optimal screening designs to D-optimal screening designs. We provide evidence that the A-optimality criterion produces more desirable screening designs than the D-optimality criterion, and that A-optimal designs generally perform better in terms of other optimality criteria than D-optimal designs. Our examples also show that, often, multiple D-optimal designs exist and that these alternative D-optimal designs can perform quite differently in terms of the A-optimality criterion. The A-optimality criterion is therefore a more discriminating optimality criterion than the D-optimality criterion.

We argue that the scale dependence of the A-optimality criterion is irrelevant due to the fact that, in screening designs, it is customary to scale each quantitative factor so that the minimum possible value is -1 and the maximum value is 1 . Also, it is customary to use effects-type coding for two-level categorical factors in screening experiments. The values -1 and 1 are then used for the two levels of the categorical factors. This puts all the factors on the same footing.

The outline of the remainder of this article is as follows. In Section 2, we discuss the two models we consider, define the optimality criteria used to construct and evaluate the screening designs in this article, and explain how to calculate relative efficiencies. In Sec- 
tion 3, we compare A- and D-optimal designs for main-effects models using three examples, in terms of four criteria, and conclude that A-optimal designs should be preferred for these models. In Section 4, we perform a similar comparison for main-effects-plus-interactions models and reach the same conclusion. In that section, we also include orthogonal arrays in two of our comparisons, as additional benchmarks. We conclude the article with a short discussion.

\section{Design optimality criteria}

\subsection{Models and estimation}

In this article, we compare A- and D-optimal designs for screening experiments. This means that we focus on main-effects models and main-effects-plus-interactions models. If we denote the number of factors by $k$ and the factors by $x_{1}, x_{2}, \ldots, x_{k}$, these models can be written as

$$
Y=\beta_{0}+\sum_{i=1}^{k} \beta_{i} x_{i}+\varepsilon
$$

and

$$
Y=\beta_{0}+\sum_{i=1}^{k} \beta_{i} x_{i}+\sum_{i=1}^{k-1} \sum_{j=i+1}^{k} \beta_{i j} x_{i} x_{j}+\varepsilon,
$$

respectively. In these expressions, $Y$ represents the response, $\beta_{0}$ is the intercept parameter, $\beta_{1}, \beta_{2}, \ldots, \beta_{k}$ are the (linear) main effects of the factors $x_{1}, x_{2}, \ldots, x_{k}, \beta_{12}, \beta_{13}, \ldots, \beta_{(k-1), k}$ are the two-factor interaction effects, and $\varepsilon$ is the normally distributed random error term. In vector notation, we write the models as

$$
Y=\mathbf{f}^{T}(\mathbf{x}) \boldsymbol{\beta}+\varepsilon
$$

where $\mathbf{f}^{T}(\mathbf{x})$ is the $p$-dimensional model expansion vector of the factors $\mathbf{x}=\left[x_{1}, x_{2}, \ldots, x_{k}\right]^{T}$, $\boldsymbol{\beta}$ is the $p$-dimensional vector containing the model parameters, and $p$ is the number of parameters in the model. For a main-effects model, $p=k+1$, while, for a main-effectsplus-interactions model, $p=(k+1)(k+2) / 2$. In matrix notation, the models can both be written as

$$
\boldsymbol{Y}=\mathrm{X} \boldsymbol{\beta}+\varepsilon
$$


where $\boldsymbol{Y}$ is the $n$-dimensional response vector, $\mathbf{X}$ is the $n \times p$ model matrix whose rows are formed by the $n$ model expansion vectors $\mathbf{f}^{T}(\mathbf{x}), \boldsymbol{\varepsilon}$ is the $n$-dimensional vector of random error terms, and $n$ denotes the number of experimental runs or observations.

Since we assume that all $n$ random errors are independent normally distributed random variables with zero mean and variance $\sigma^{2}$, the best linear unbiased estimator for the parameter vector $\boldsymbol{\beta}$ is the ordinary least squares estimator

$$
\hat{\boldsymbol{\beta}}=\left(\mathbf{X}^{T} \mathbf{X}\right)^{-1} \mathbf{X}^{T} \boldsymbol{Y}
$$

The variance-covariance matrix of this estimator is

$$
\sigma^{2}\left(\mathbf{X}^{T} \mathbf{X}\right)^{-1}
$$

The inverse of that matrix,

$$
\sigma^{-2}\left(\mathbf{X}^{T} \mathbf{X}\right)
$$

is the Fisher information matrix and summarizes the information content of the experimental design concerning the parameters in the model under investigation.

In this article, we focus on the choice of an optimal screening design for a given number of runs $n$. Since the value of $\sigma^{2}$ is unimportant in that scenario, we set it to 1 without loss of generality. For this reason, all variances and standard errors we report in this article are relative to $\sigma^{2}$ and $\sigma$, respectively. Because our focus is on screening experiments, we construct A- and D-optimal designs in this article. This is because experimenters desire powerful significance tests in screening experimentation and precise estimates of the model parameters yield large powers for such tests. The best-known optimality criteria that focus on precise parameter estimates are the A- and D-optimality criteria. We compare the Aand D-optimal designs in terms of the A-, D-, G- and I-optimality criteria.

\subsection{Optimality criteria}

In this section, we define the four optimality criteria we use in this article. The definitions of the A-, D- and G-optimality criteria do not depend on the nature of the factors under study. The I-optimality criterion depends on whether the experimental factors are quantitative or two-level categorical factors. In all of our examples, we treat the factors as quantitative, because this is usually where the differences between A- and D-optimal designs occur. 


\subsubsection{A-optimality}

An A-optimal design minimizes the average variance of the $p$ parameter estimates in $\hat{\boldsymbol{\beta}}$. As a result, an A-optimal design minimizes the sum of the diagonal elements of the ordinary least squares estimator's variance-covariance matrix. Since that sum is called the trace of the variance-covariance matrix, an A-optimal design minimizes trace $\left(\mathbf{X}^{T} \mathbf{X}\right)^{-1}$.

In this article, we use the relative A-efficiency to compare the quality of two designs with model matrices $\mathbf{X}_{1}$ and $\mathbf{X}_{2}$ in terms of the A-optimality criterion. The relative Aefficiency of a design with model matrix $\mathbf{X}_{1}$ with respect to a design with model matrix $\mathbf{X}_{2}$ is defined as

$$
\frac{\operatorname{trace}\left(\mathbf{X}_{2}^{T} \mathbf{X}_{2}\right)^{-1}}{\operatorname{trace}\left(\mathbf{X}_{1}^{T} \mathbf{X}_{1}\right)^{-1}} \text {. }
$$

A relative A-efficiency larger than one indicates that the former design is better than the latter in terms of A-optimality.

\subsubsection{D-optimality}

A D-optimal design maximizes the determinant of the Fisher information matrix, $\left|\mathbf{X}^{T} \mathbf{X}\right|$. Since this determinant is inversely related to the volume of the $p$-dimensional confidence ellipsoid about the parameters in $\boldsymbol{\beta}$, a D-optimal design minimizes that volume for a given number of runs $n$.

We use the relative D-efficiency to compare the quality of two designs with model matrices $\mathbf{X}_{1}$ and $\mathbf{X}_{2}$ in terms of the D-optimality criterion. The relative D-efficiency of a design with model matrix $\mathbf{X}_{1}$ with respect to a design with model matrix $\mathbf{X}_{2}$ is defined as $\left(\left|\mathbf{X}_{1}^{T} \mathbf{X}_{1}\right| /\left|\mathbf{X}_{2}^{T} \mathbf{X}_{2}\right|\right)^{1 / p}$. A relative D-efficiency larger than one indicates that the former design is better than the latter in terms of the D-optimality criterion.

\subsubsection{I-optimality}

An I-optimal design minimizes the average variance of prediction over all possible factor level combinations. In the event all $k$ experimental factors are quantitative, the average variance of prediction can be expressed as

$$
\frac{\int_{\chi} \mathbf{f}^{T}(\mathbf{x})\left(\mathbf{X}^{T} \mathbf{X}\right)^{-1} \mathbf{f}(\mathbf{x}) d \mathbf{x}}{\int_{\chi} d \mathbf{x}}
$$


where $\chi$ denotes the experimental region. As pointed out in Goos and Jones (2011), when all experimental factors are quantitative, we can calculate the average prediction variance as

$$
\frac{1}{\int_{\chi} d \mathbf{x}} \cdot \operatorname{tr}\left[\left(\mathbf{X}^{T} \mathbf{X}\right)^{-1} \mathbf{B}\right]
$$

where

$$
\mathbf{B}=\int_{\chi} \mathbf{f}(\mathbf{x}) \mathbf{f}^{T}(\mathbf{x}) d \mathbf{x}
$$

This $p$-dimensional matrix is called the moments matrix because its elements are proportional to moments of a uniform distribution on the experimental region $\chi$. Assuming the experimental region $\chi$ is $[-1,1]^{k}$,

$$
\mathbf{B}=2^{k} \operatorname{diag}\left(1,1 / 3 \mathbf{I}_{k}\right)
$$

in the event a main-effects model is used, and

$$
\mathbf{B}=2^{k} \operatorname{diag}\left(1,1 / 3 \mathbf{I}_{k}, 1 / 9 \mathbf{I}_{k(k-1) / 2}\right)
$$

in the event a main-effects-plus-interactions model is used. In these expressions, $\mathbf{I}_{k}$ and $\mathbf{I}_{k(k-1) / 2}$ are $k$ - and $k(k-1) / 2$-dimensional identity matrices, respectively. The first diagonal element of the moments matrices corresponds to the intercept, while the next $k$ diagonal elements correspond to the main effects.

In the event all $k$ factors are two-level categorical factors, the average variance of prediction equals

$$
\frac{\sum_{\chi} \mathbf{f}^{T}(\mathbf{x})\left(\mathbf{X}^{T} \mathbf{X}\right)^{-1} \mathbf{f}(\mathbf{x}) d \mathbf{x}}{2^{k}}=2^{-k} \operatorname{tr}\left[\left(\mathbf{X}^{T} \mathbf{X}\right)^{-1} \mathbf{B}\right],
$$

where the sum is over all $2^{k}$ possible test combinations, and the moments matrix $\mathbf{B}$ is the $2^{k}$ times the $p$-dimensional identity matrix. Therefore, the I-optimality criterion is equivalent to the A-optimality criterion if all $k$ factors are two-level categorical ones.

Also for main-effects and main-effects-plus-interactions models involving $k$ quantitative factors, the I-optimality criterion and the A-optimality criterion are close. If the diagonal elements of the moments matrix $\mathbf{B}$ were all equal, then the two criteria would be equivalent for quantitative factors as well. However, for main-effects models, compared to the Aoptimality criterion, the I-optimality criterion attaches a larger importance to the variance of the intercept estimate. This is due to the fact that the first diagonal element of $\mathbf{B}$ is 
larger than the other diagonal elements. For main-effects-plus-interactions models, the Ioptimality criterion attaches a larger importance to the variance of the intercept estimate and a smaller importance to the variances of the estimates of the interactions. This is due to the facts that (i) the first diagonal element of $\mathbf{B}$ is larger than the diagonal elements corresponding to the main effects and (ii) the last $k(k-1) / 2$ diagonal elements are smaller than those corresponding to the main effects. Due to the resemblance of the A- and Ioptimality criteria for the models considered in this article, we expect experimental designs that perform well in terms of the A-optimality criterion to also perform well in terms of the I-optimality criteron.

To compare the performance of two designs with model matrices $\mathbf{X}_{1}$ and $\mathbf{X}_{2}$ in terms of the I-optimality criterion, we use the relative I-efficiency. The relative I-efficiency of a design with model matrix $\mathbf{X}_{1}$ with respect to a design with model matrix $\mathbf{X}_{2}$ is

$$
\frac{\operatorname{trace}\left[\left(\mathbf{X}_{2}^{T} \mathbf{X}_{2}\right)^{-1} \mathbf{B}\right]}{\operatorname{trace}\left[\left(\mathbf{X}_{1}^{T} \mathbf{X}_{1}\right)^{-1} \mathbf{B}\right]}
$$

A relative I-efficiency larger than one indicates that the former design is better than the latter in terms of I-optimality.

\subsubsection{G-optimality}

A G-optimal design minimizes the maximum variance of prediction over the experimental region $\chi$,

$$
\max _{\mathbf{x} \in \chi} \mathbf{f}^{T}(\mathbf{x})\left(\mathbf{X}^{T} \mathbf{X}\right)^{-1} \mathbf{f}(\mathbf{x})
$$

To compare the performance of two designs with model matrices $\mathbf{X}_{1}$ and $\mathbf{X}_{2}$ in terms of the G-optimality criterion, we use the relative G-efficiency. The relative G-efficiency of a design with model matrix $\mathbf{X}_{1}$ with respect to a design with model matrix $\mathbf{X}_{2}$ is

$$
\frac{\max _{\mathbf{x} \in \chi} \mathbf{f}^{T}(\mathbf{x})\left(\mathbf{X}_{2}^{T} \mathbf{X}_{2}\right)^{-1} \mathbf{f}(\mathbf{x})}{\max _{\mathbf{x} \in \chi} \mathbf{f}^{T}(\mathbf{x})\left(\mathbf{X}_{1}^{T} \mathbf{X}_{1}\right)^{-1} \mathbf{f}(\mathbf{x})} .
$$

A relative G-efficiency larger than one indicates that the former design is better than the latter in terms of G-optimality. 


\section{Main-effects model}

\section{$3.1 \quad$ General}

\subsubsection{Run size is a multiple of 4}

For run sizes $n$ that are multiples of four (i.e., $n \equiv 0 \bmod 4$ ), all D- and A-optimal designs for main-effects models are two-level orthogonal designs. The best known of these designs are Plackett-Burman designs, but, depending on the run size, there may be many other orthogonal two-level designs (see, for instance, Schoen et al. (2017)).

\subsubsection{Run size is a multiple of 4 plus 1}

When the run size $n$ is one more than a multiple of 4 (i.e., $n \equiv 1 \bmod 4$ ), D- and Aoptimal designs for main-effects models coincide as well and also involve two levels for each factor. For $n>k+1$, this was proven by Cheng (1980). For the saturated case, where the number of runs equals the number of model parameters and thus $n=k+1$, Cheng (1980) proved that the D- and A-optimal designs coincide and involve two levels per factor as well whenever $2 n-1$ is a squared integer (i.e., when $n$ is $5,13,25,41, \ldots$ ). Our computational results suggest that D- and A-optimal saturated designs also coincide and only involve two factor levels for $n$ values of $9,17,21,29,33$ and 37. We could not find any formal proof of this in the literature.

In conclusion, for main-effects models and run sizes that are one more than a multiple of 4, we conjecture that A-optimal designs do not differ from D-optimal designs, and involve two levels for each factor.

\subsubsection{Run size is a multiple of 4 plus 2}

For run sizes $n$ that are odd multiples of 2 (i.e., $n \equiv 2 \bmod 4$ ), Jacroux et al. (1983) proved that, within the family of two-level experimental designs, D- and A-optimal designs are equivalent. The results of Wong and Masaro (1984) suggest that, for small values of the run size $n$, the true A-optimal designs might involve three levels for at least one factor. Our computational results, however, do not provide support for this claim. Therefore, our computational experience suggests that, also when $n$ is an odd multiple of 2, A-optimal 
designs for main-effects models do not differ from D-optimal designs, and involve two factor levels only.

\subsubsection{Run size is a multiple of 4 plus 3}

The only run sizes where differences can occur between D- and A-optimal designs for maineffects models are those that are one less than multiples of four (i.e., $n \equiv 3 \bmod 4$ ). Remarkably, some of the A-optimal designs with these run sizes involve the zero level for one or more factors. A-optimal screening designs can therefore be three-level designs rather than two-level designs. Several examples of A-optimal designs involving three levels for one of the factors were provided by Wong and Masaro (1984). We systematically compared Aand D-optimal designs with numbers of factors ranging from 3 to 15 and numbers of runs up to 23 , and observed differences when the number of runs is relatively small compared to the number of factors. For instance, for three factors, the 7-run A- and D-optimal designs differ, but the 11-run A- and D-optimal designs are equal. For four factors, the 7- and 11run A- and D-optimal designs differ, but not the 15-run designs. For five and six factors, the 7-, 11- and 15-run A- and D-optimal designs differ, but not the 19-run designs. For seven factors, the 11-, 15- and 19-run A- and D-optimal designs differ. For eight, nine and ten factors, the 11-, 15-, 19- and 23-run A- and D-optimal designs differ. For 11-14 factors, the 15-, 19- and 23-run A- and D-optimal designs differ. Finally, the 19- and 23-run Aand D-optimal designs differ for 15 factors.

Galil and Kiefer $(1980,1982)$ indicated that, within the family of two-level designs, Dand A-optimal designs may differ when $n=3 \bmod 4$. The differences between A-optimal and D-optimal designs can occur only when the run size $n$ is smaller than a certain upper bound that is a highly complex function of the number of model parameters (Masaro; 1983). When there are at least three factors, that upper bound can be tightened to

$$
\frac{1}{4}(7 p-16+\sqrt{(p-4)(17 p-36)})
$$

as was shown by Sathe and Shenoy (1989). 
Table 1: A- and D-optimal 7-run, 4-factor designs for the main-effects model

\begin{tabular}{rrrr|rrrr}
\hline \multicolumn{3}{c}{ A-optimal } & \multicolumn{5}{c}{ D-optimal } \\
\hline$x_{1}$ & $x_{2}$ & $x_{3}$ & $x_{4}$ & $x_{1}$ & $x_{2}$ & $x_{3}$ & $x_{4}$ \\
\hline 1 & -1 & 1 & 0 & 1 & 1 & 1 & 1 \\
1 & 1 & -1 & 1 & 1 & 1 & 1 & -1 \\
-1 & -1 & -1 & 1 & 1 & -1 & -1 & 1 \\
-1 & -1 & -1 & -1 & 1 & -1 & -1 & -1 \\
-1 & 1 & 1 & 1 & -1 & 1 & -1 & 1 \\
1 & 1 & -1 & -1 & -1 & 1 & -1 & -1 \\
-1 & 1 & 1 & -1 & -1 & -1 & 1 & 1 \\
\hline
\end{tabular}

\subsection{Examples}

In the remainder of Section 3, we present three examples of cases where the A- and Doptimal designs differ. Because the only run sizes for which differences occur between Aand D-optimal designs are multiples of four plus 3 , the run sizes in the examples are 7,11 and 15. In two of the examples, there are several residual degrees of freedom when fitting the main-effects model. The third example is saturated (i.e., $n=k+1$ ). We use that example not because we recommend the use of saturated designs, but because it is a case for which various D-optimal designs have been reported in the literature.

\subsubsection{Example 1: 7 runs and 4 factors}

Consider the problem of designing an experiment involving seven runs to estimate the main effects of four quantitative factors. The traditional D-optimal designs for this problem are two-level designs involving seven of the 16 vertices of the experimental region. The Aoptimal designs only involve six of these vertices. The seventh run of any given A-optimal design is the centroid of one of the experimental region's edges. So, in that run, one of the four factors acts at its middle level. An A-optimal design and a D-optimal design produced by JMP are shown in Table 1. In the A-optimal design shown in the table, the first row involves the middle level for the fourth factor. 

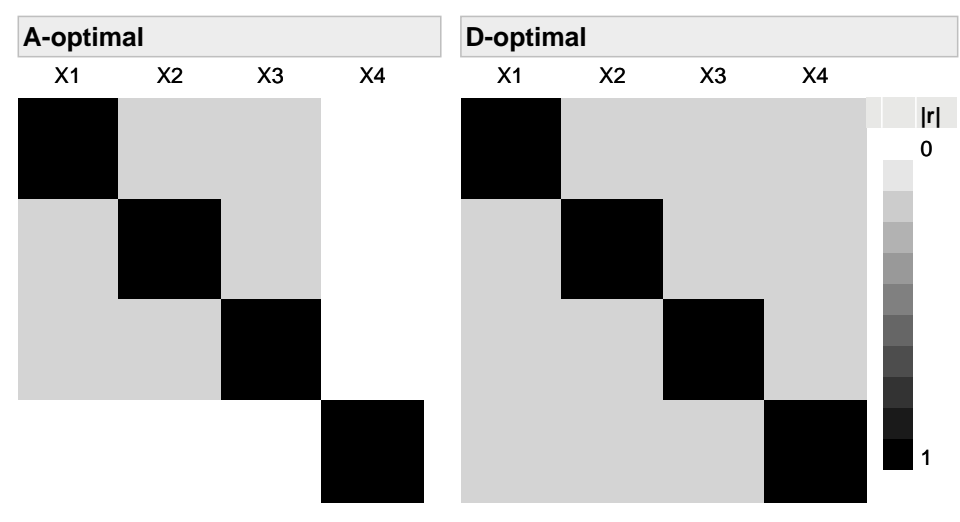

Figure 1: Color maps of the correlations between the main-effects columns of the A- and D-optimal 7-run, 4-factor designs in Table 1.

The fact that the A-optimal design involves the middle level of $x_{4}$ in one of the runs implies that only six of its seven runs provide information concerning the main effect of $x_{4}$. In the D-optimal design, none of the seven runs involves a zero for $x_{4}$, so all seven runs provide information concerning the main effect of $x_{4}$. Nevertheless, the precision of the estimate for $x_{4}$ 's main effect is equal for both designs. To understand this, it is useful to study the color maps of the correlations between the main-effects columns of the model matrices corresponding to the two designs, shown in Figure 1. All the off-diagonal cells in the color map for the D-optimal design are grey, indicating that every pair of main-effects columns are correlated. The absolute correlations all equal $1 / 6$. The color map for the A-optimal design shows that the main-effect column for $x_{4}$ is uncorrelated with the other columns, indicating that $x_{4}$ 's main effect can be estimated independently. The three other main-effects columns are correlated, as in the D-optimal design. The absolute correlations for these main-effects columns are $1 / 6$ as well. It is due to the multicollinearity involving the column for $x_{4}$ that the D-optimal design does not allow a more precise estimation of $x_{4}$ 's main effect than the A-optimal design.

That the A-optimal design involves less multicollinearity than the D-optimal design is also visible in the designs' information matrices. For the D-optimal design, the information 
matrix equals

$$
\left[\begin{array}{rrrrr}
7 & 1 & 1 & -1 & 1 \\
1 & 7 & -1 & 1 & -1 \\
1 & -1 & 7 & 1 & -1 \\
-1 & 1 & 1 & 7 & 1 \\
1 & -1 & -1 & 1 & 7
\end{array}\right],
$$

while, for the A-optimal design, it is

$$
\left[\begin{array}{rrrrr}
7 & -1 & 1 & -1 & 0 \\
-1 & 7 & 1 & -1 & 0 \\
1 & 1 & 7 & 1 & 0 \\
-1 & -1 & 1 & 7 & 0 \\
0 & 0 & 0 & 0 & 6
\end{array}\right] .
$$

The specific structure of the D-optimal design implies that the relative standard error for all model parameters is $\sqrt{1 / 6}=0.40825$. The A-optimal design leads to smaller standard errors of 0.39529 for the intercept and for the main effects of $x_{1}, x_{2}$ and $x_{3}$, and to the same standard error of $\sqrt{1 / 6}=0.40825$ for the main effect of $x_{4}$. So, for four of the five model parameters, the A-optimal design produces standard errors that are $3.3 \%$ lower than does the D-optimal design. For the fifth model parameter, the standard errors are the same. The more precise estimates produced by the A-optimal design cause it to be $5.3 \%$ more A-efficient than the D-optimal design.

Remarkably, the relative D-efficiency of the A-optimal design with respect to the Doptimal design is $100 \%$. So, the A-optimal design is D-optimal for the main-effects model too, despite the fact that it is not a two-level design. To many, this will be a surprising result because, when searching for D-optimal designs for main-effects models involving quantitative factors, it suffices to consider two levels per factor. This is due to the fact that there always exists a D-optimal design with two levels per factor. So, considering a third level is unnecessary for finding D-optimal designs for main-effects models (Box and Draper; 1971; Mitchell; 1974; Galil and Kiefer; 1980).

In terms of prediction variance, the A-optimal design outperforms the D-optimal design. First, the A-optimal design is $5.7 \%$ better than the D-optimal design in terms of I-efficiency. 
Second, it is $42.9 \%$ better in terms of G-efficiency. This is due to the fact that, for the A-optimal design, the maximum prediction variance is $7 / 6$ (that maximum is reached at $\left.\left(x_{1}, x_{2}, x_{3}, x_{4}\right)=(1,-1,1,-1)\right)$, while, for the D-optimal design, the maximum prediction variance is $5 / 3$ (that maximum is reached at $\left(x_{1}, x_{2}, x_{3}, x_{4}\right)=(-1,-1,1,-1)$ ). Therefore, the relative G-efficiency for the A-optimal design compared to the D-optimal design is $(5 / 3) /(7 / 6)=10 / 7$.

\subsubsection{Example 2: 15 runs and 9 factors}

Our second example is concerned with the problem of designing an experiment involving 15 runs to estimate the main effects of nine quantitative factors. The traditional D-optimal designs again have two levels for each of the factors. The A-optimal designs, however, involve three levels for two of the nine factors. One A-optimal design we obtained using JMP is shown in Table 2. The eighth run in that design uses the middle level for both $x_{8}$ and $x_{9}$. A D-optimal design we obtained using JMP is shown in Table 3.

The effect of the two zeroes for the color map of the correlations between the maineffects columns of the model matrix is shown in Figure 2. Fourteen of the off-diagonal cells in the A-optimal design's color map are white, meaning that 14 pairs of main-effects columns are uncorrelated. In contrast, the D-optimal design has no uncorrelated pairs of main-effects columns. All of the 36 column pairs for the D-optimal design have absolute correlations of $1 / 14$. The A-optimal design has 21 pairs of columns exhibiting that absolute correlation, and one pair with an absolute correlation of $1 / 7$ (namely, the columns for $x_{8}$ and $x_{9}$ ). So, the total amount of multicollinearity is smaller in the A-optimal design than in the D-optimal design.

The smaller amount of multicollinearity in the A-optimal design results in more precise estimates for seven of the nine main effects (i.e., for the main effects of the first seven factors) and for the intercept. For the main effects of $x_{8}$ and $x_{9}$, there is no difference in precision between the two designs. As a result, the A-efficiency of the A-optimal design is $2.9 \%$ better than that of the two-level D-optimal design in Table 3.

Remarkably, the A-optimal design shown in Table 2 and involving three levels for $x_{8}$ and $x_{9}$ turns out to be D-optimal as well: its D-efficiency relative to the two-level D-optimal 
Table 2: A-optimal 15-run, 9-factor design for the main-effects model

\begin{tabular}{rrrrrrrrr}
\hline$x_{1}$ & $x_{2}$ & $x_{3}$ & $x_{4}$ & $x_{5}$ & $x_{6}$ & $x_{7}$ & $x_{8}$ & $x_{9}$ \\
\hline 1 & 1 & 1 & 1 & 1 & 1 & 1 & 1 & 1 \\
-1 & 1 & -1 & 1 & -1 & 1 & -1 & 1 & -1 \\
1 & -1 & -1 & 1 & 1 & -1 & -1 & 1 & 1 \\
-1 & -1 & 1 & 1 & -1 & -1 & 1 & 1 & -1 \\
1 & 1 & 1 & -1 & -1 & -1 & -1 & 1 & 1 \\
-1 & 1 & -1 & -1 & 1 & -1 & 1 & 1 & -1 \\
1 & -1 & -1 & -1 & -1 & 1 & 1 & 1 & 1 \\
-1 & -1 & 1 & -1 & 1 & 1 & -1 & 0 & 0 \\
1 & 1 & 1 & 1 & 1 & 1 & 1 & -1 & -1 \\
-1 & 1 & -1 & 1 & -1 & 1 & -1 & -1 & 1 \\
1 & -1 & -1 & 1 & 1 & -1 & -1 & -1 & -1 \\
-1 & -1 & 1 & 1 & -1 & -1 & 1 & -1 & 1 \\
1 & 1 & 1 & -1 & -1 & -1 & -1 & -1 & -1 \\
-1 & 1 & -1 & -1 & 1 & -1 & 1 & -1 & 1 \\
1 & -1 & -1 & -1 & -1 & 1 & 1 & -1 & -1 \\
\hline
\end{tabular}

design we obtained from JMP is $100 \%$. So, in this second example, we again obtain the surprising result that a three-level design is D-optimal for a main-effects model.

The A-optimal design in Table 2 is $3.1 \%$ better than the D-optimal one in terms of I-efficiency, and it is $42.9 \%$ better in terms of G-efficiency. For the A-optimal design, the maximum prediction variance again amounts to $7 / 6$, and, for the D-optimal design, the maximum prediction variance is again $5 / 3$.

\subsection{Example 3: 11 runs and 10 factors}

In this section, we compare two 11-run designs for estimating a 10-factor main-effects model. One of the designs is D-optimal. It involves two levels for each factor and is shown in Table 4. Of five different D-optimal designs we encountered for this example, 
Table 3: D-optimal 15-run, 9-factor design for the main-effects model

\begin{tabular}{rrrrrrrrr}
\hline$x_{1}$ & $x_{2}$ & $x_{3}$ & $x_{4}$ & $x_{5}$ & $x_{6}$ & $x_{7}$ & $x_{8}$ & $x_{9}$ \\
\hline-1 & 1 & -1 & 1 & -1 & 1 & -1 & 1 & -1 \\
1 & -1 & -1 & 1 & 1 & -1 & -1 & 1 & 1 \\
-1 & -1 & 1 & 1 & -1 & -1 & 1 & 1 & -1 \\
1 & 1 & 1 & -1 & -1 & -1 & -1 & 1 & 1 \\
-1 & 1 & -1 & -1 & 1 & -1 & 1 & 1 & -1 \\
1 & -1 & -1 & -1 & -1 & 1 & 1 & 1 & 1 \\
-1 & -1 & 1 & -1 & 1 & 1 & -1 & 1 & -1 \\
1 & 1 & 1 & 1 & 1 & 1 & 1 & -1 & -1 \\
-1 & 1 & -1 & 1 & -1 & 1 & -1 & -1 & 1 \\
1 & -1 & -1 & 1 & 1 & -1 & -1 & -1 & -1 \\
-1 & -1 & 1 & 1 & -1 & -1 & 1 & -1 & 1 \\
1 & 1 & 1 & -1 & -1 & -1 & -1 & -1 & -1 \\
-1 & 1 & -1 & -1 & 1 & -1 & 1 & -1 & 1 \\
1 & -1 & -1 & -1 & -1 & 1 & 1 & -1 & -1 \\
-1 & -1 & 1 & -1 & 1 & 1 & -1 & -1 & 1 \\
\hline
\end{tabular}

that design performed best in terms of A-optimality. It was, for example, better in terms of A-optimality than the 11-run 10-factor main-effects designs reported in Galil and Kiefer (1980) and constructed by Ehlich and Zeller (1962). The second design in our comparison, shown in Table 5, is an A-optimal design we obtained using JMP. The A-optimal design in Table 5 has four zero entries. Two of these zero entries appear in the design's first column. The remaining two zeroes appear in the design's last row.

Due to its four zeroes, the A-optimal design has 13 pairs of uncorrelated main-effects columns. This can be seen in the design's color map in Figure 3. The design also has three pairs of main-effects columns with the maximum absolute correlation of 0.2667 . The D-optimal design has no uncorrelated pairs of main-effects columns. Its minimum absolute correlation is 0.0690 (which occurs for 15 pairs of main-effects columns). It has an absolute correlation of 0.2143 for six pairs of main-effects columns, an absolute correlation of 0.1 


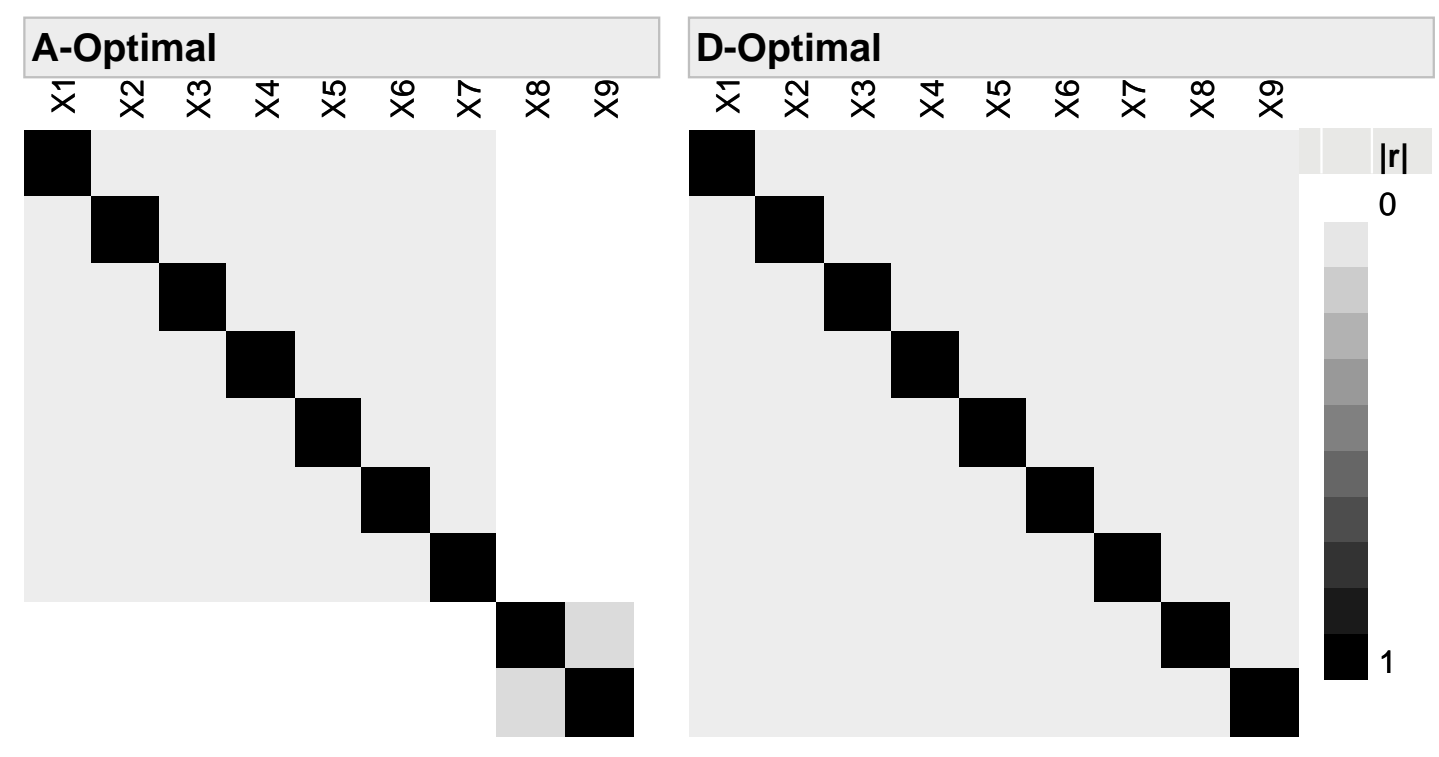

Figure 2: Color maps of the correlations between the main-effects columns of the A- and D-optimal 15-run, 9-factor designs discussed in Section 3.2.2.

for twelve pairs and an absolute correlation of 0.2667 for three pairs. The average absolute correlation across all pairs of main-effects columns is 0.0814 for the A-optimal design, and 0.1098 for the D-optimal design. So, the A-optimal design involves less multicollinearity than the D-optimal design.

Eight of the 10 main-effect estimates are more precise when using the A-optimal design than when using the D-optimal design. For the two remaining main-effects, the estimates obtained from the D-optimal designs are more precise. One main effect that is estimated more precisely using the D-optimal designs is that of factor $x_{1}$, which is the factor that has two zero levels in the design. The other is the main effect of $x_{6}$, which is the second factor that has a single zero in the design. The fact that the A-optimal design provides better estimates for eight of the main effects, and for the intercept, causes it to be more A-efficient than the D-optimal design. This is shown in Table 6, where we also display the relative D-, G- and I-efficiencies. This time, the A-optimal design is not D-optimal, since its relative D-efficiency is slightly smaller than one. The A-optimal design has a slightly higher I-efficiency than the D-optimal design. Finally, it is about 3\% less G-efficient than the D-optimal design. 
Table 4: D-optimal 11-run, 10-factor design for the main-effects model, which is A-optimal within the class of two-level designs.

\begin{tabular}{rrrrrrrrrr}
\hline$x_{1}$ & $x_{2}$ & $x_{3}$ & $x_{4}$ & $x_{5}$ & $x_{6}$ & $x_{7}$ & $x_{8}$ & $x_{9}$ & $x_{10}$ \\
\hline 1 & 1 & 1 & 1 & 1 & 1 & 1 & 1 & 1 & 1 \\
1 & 1 & 1 & 1 & 1 & 1 & -1 & -1 & -1 & -1 \\
1 & 1 & 1 & 1 & -1 & -1 & 1 & 1 & -1 & -1 \\
1 & 1 & 1 & 1 & -1 & -1 & -1 & -1 & 1 & 1 \\
1 & 1 & 1 & -1 & -1 & 1 & -1 & 1 & -1 & 1 \\
1 & -1 & -1 & 1 & 1 & -1 & -1 & 1 & -1 & 1 \\
1 & -1 & -1 & -1 & -1 & 1 & 1 & -1 & 1 & -1 \\
-1 & 1 & -1 & 1 & -1 & 1 & -1 & 1 & 1 & -1 \\
-1 & 1 & -1 & -1 & 1 & -1 & 1 & -1 & -1 & 1 \\
-1 & -1 & 1 & 1 & -1 & 1 & 1 & -1 & -1 & 1 \\
-1 & -1 & 1 & -1 & 1 & -1 & -1 & 1 & 1 & -1 \\
\hline
\end{tabular}

It is noteworthy that the D-optimal design in Table 4 is A-optimal within the class of two-level designs. Allowing for more than two levels per factor when searching for Aoptimal designs thus leads to a slightly higher A-efficiency: the A-optimal design in Table 5 is $0.7 \%$ more A-efficient than the design in Table 4 .

Finally, among the various D-optimal designs we encountered for this example, the maximum difference in A-efficiency was 2.8\%. The maximum difference in I-efficiency was $1.7 \%$, and the difference in G-efficiency was $38.2 \%$. Considering this difference in Gefficiency and the difference in the correlation patterns in the color maps in Figure 3, this example confirms that there can be large differences between D- and A-optimal designs in certain properties, in favor of the A-optimal design. 
Table 5: A-optimal 11-run, 10-factor design for the main-effects model

\begin{tabular}{rrrrrrrrrr}
\hline$x_{1}$ & $x_{2}$ & $x_{3}$ & $x_{4}$ & $x_{5}$ & $x_{6}$ & $x_{7}$ & $x_{8}$ & $x_{9}$ & $x_{10}$ \\
\hline 0 & -1 & -1 & 1 & 1 & 1 & -1 & 1 & 1 & -1 \\
-1 & -1 & -1 & -1 & -1 & -1 & -1 & -1 & -1 & -1 \\
0 & 1 & 1 & -1 & 1 & -1 & -1 & -1 & 1 & 1 \\
-1 & 1 & -1 & 1 & -1 & 1 & 1 & -1 & 1 & 1 \\
1 & -1 & 1 & 1 & -1 & 1 & -1 & -1 & -1 & -1 \\
1 & -1 & -1 & -1 & 1 & 1 & 1 & -1 & -1 & 1 \\
-1 & -1 & 1 & 1 & 1 & -1 & 1 & 1 & -1 & 1 \\
1 & 1 & -1 & 1 & 1 & -1 & 1 & -1 & -1 & -1 \\
-1 & 1 & 1 & -1 & 1 & 1 & 1 & 1 & -1 & -1 \\
1 & -1 & 1 & -1 & -1 & -1 & 1 & 1 & 1 & -1 \\
1 & 1 & -1 & 0 & -1 & 0 & -1 & 1 & -1 & 1 \\
\hline
\end{tabular}

\section{Main-effects-plus-interactions model}

\subsection{General}

There exist no theoretical results concerning A-optimal designs for estimating all main effects and all two-factor interactions. The only scenarios in which A-optimal designs are known in that case are when full factorial two-level designs, resolution- $V$ fractional factorial two-level designs, and strength-4 orthogonal arrays can be used. When these

Table 6: Efficiencies of the A-optimal 11-run 10-factor design in Table 5 relative to the D-optimal design in Table 4.

\begin{tabular}{cc}
\hline Criterion & Efficiency \\
\hline D & 0.995 \\
G & 0.971 \\
A & 1.007 \\
I & 1.018 \\
\hline
\end{tabular}




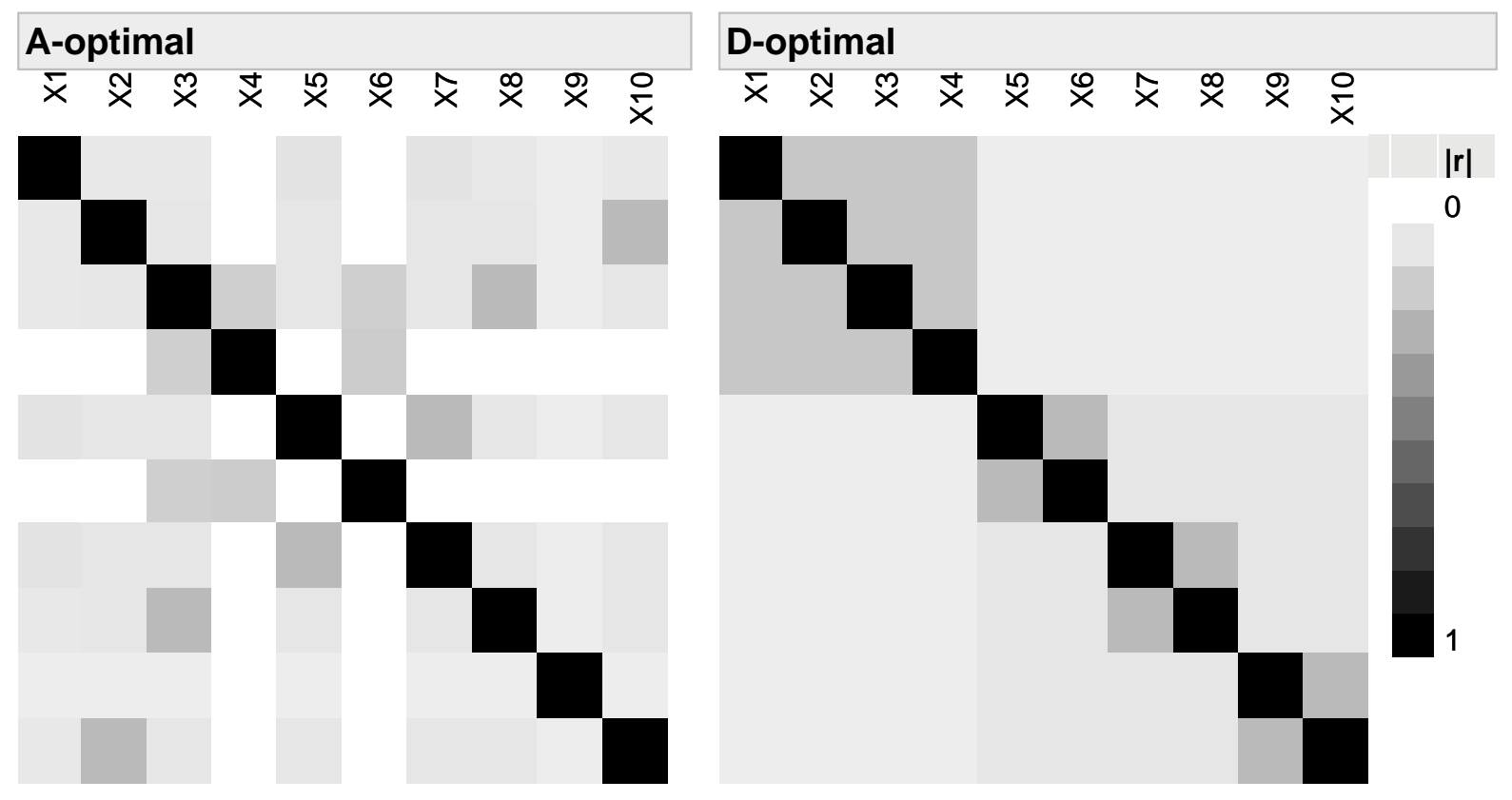

Figure 3: Color maps of the correlations between the main-effects columns of the D- and A-optimal 11-run, 10-factor designs shown in Tables 4 and 5, respectively.

designs are used, the information matrix for a model with intercept, main effects and twofactor interactions is equal to $n \mathbf{I}_{p}$ and the main effects and interactions can be independently estimated with maximal precision. As a result, A-optimal designs are known for maineffects-plus-interactions models only when the number of runs is large and a multiple of 4 . In all those cases, the A-optimal designs are D-optimal as well.

For main-effects-plus-interactions models, we systematically compared A- and D-optimal designs with numbers of factors ranging from 3 to 7 and numbers of runs up to 31 . For three factors, the 7-run A- and D-optimal designs differ, but the A- and D-optimal designs are equal for any run size larger than 7 . For four factors, A- and D-optimal designs differ for run sizes ranging from 11 to 15. For five factors, we could not find any difference between A- and D-optimal designs. For six factors, we found differences between A- and D-optimal designs for run sizes ranging from 27 to 31. Finally, for seven factors, we found differences between the 31-run A-optimal design and its D-optimal counterpart. In summary, there are again multiple cases where A- and D-optimal designs exist. Unlike for the main-effects 
model, A- and D-optimal designs can differ regardless of whether the run size is a multiple of four, one more, two more or three more.

In the remainder of this section, we discuss two examples involving differences between the D- and A-optimal designs. The first example involves a nearly saturated example, involving the commonly used run size 12 (due to the existence of a Plackett-Burman design with that run size). The second example involves several residual degrees of freedom and allows us to pick a benchmark orthogonal array from the literature.

\subsection{Examples}

\subsubsection{Example 4: 12 runs and 4 factors}

Our first main-effects-plus-interactions example involves 12 runs and four factors. We compare three designs. The first design is the A-optimal design shown in the left panel of Table 7 and obtained using JMP. One of the factors in that design, $x_{1}$, is studied at three levels, while the other three have only two levels. The A-optimal design is balanced in the three levels used for $x_{1}$, so there are four zero entries in the $x_{1}$ column. The second design under comparison is a D-optimal design produced by JMP as well. That design, which is shown in the middle panel of Table 7, studies each factor at two levels, and turns out to be A-optimal for the main-effects-plus-interactions model within the class of two-level designs. The third design under comparison is a 12-run 4-factor two-level orthogonal array, which is equivalent to the design obtained by dropping seven columns from the 12-run 11-factor Plackett-Burman design and which is commonly used in practice. That design is shown in the rightmost panel of Table 7 .

The color maps showing the correlations between the main-effects and two-factorinteraction columns for the three designs are displayed in Figure 4. The color map for the A-optimal design shows that there are only three pairs of columns in its model matrix that have nonzero correlations. These nonzero correlations all equal $1 / 3$ in absolute value. The D-optimal design has substantially fewer zero correlations between pairs of columns from its model matrix. It also involves three absolute correlations of $1 / 3$ and 24 pairs with an absolute correlation of 0.1690 . The orthogonal array is orthogonal for the main effects, but it involves 15 pairs of columns in the model matrix that have an absolute cor- 
Table 7: A-optimal, D-optimal and orthogonal-array-based 12-run, 4-factor designs for the main-effects-plus-interactions model

\begin{tabular}{rrrr|rrrr|rrrr}
\hline \multicolumn{4}{c}{ A-optimal } & \multicolumn{4}{c}{ D-optimal } & \multicolumn{3}{c}{ Orthogonal array } \\
\hline$x_{1}$ & $x_{2}$ & $x_{3}$ & $x_{4}$ & $x_{1}$ & $x_{2}$ & $x_{3}$ & $x_{4}$ & $x_{1}$ & $x_{2}$ & $x_{3}$ & $x_{4}$ \\
\hline 0 & -1 & 1 & -1 & -1 & 1 & 1 & -1 & -1 & 1 & 1 & 1 \\
-1 & -1 & 1 & 1 & 1 & 1 & 1 & -1 & 1 & 1 & 1 & -1 \\
1 & 1 & -1 & 1 & 1 & -1 & 1 & 1 & -1 & 1 & 1 & -1 \\
0 & 1 & 1 & 1 & -1 & -1 & 1 & -1 & 1 & 1 & -1 & 1 \\
1 & 1 & 1 & -1 & 1 & -1 & 1 & -1 & 1 & -1 & 1 & 1 \\
1 & -1 & -1 & -1 & -1 & -1 & 1 & 1 & -1 & -1 & -1 & -1 \\
-1 & -1 & -1 & -1 & -1 & -1 & -1 & 1 & 1 & -1 & -1 & -1 \\
-1 & 1 & 1 & -1 & -1 & 1 & -1 & 1 & 1 & -1 & 1 & -1 \\
0 & -1 & -1 & 1 & -1 & 1 & -1 & -1 & -1 & -1 & -1 & 1 \\
0 & 1 & -1 & -1 & 1 & -1 & -1 & -1 & -1 & 1 & -1 & -1 \\
-1 & 1 & -1 & 1 & 1 & 1 & 1 & 1 & -1 & -1 & 1 & 1 \\
1 & -1 & 1 & 1 & 1 & 1 & -1 & 1 & 1 & 1 & -1 & 1 \\
\hline
\end{tabular}

relation of $1 / 3$. So, in terms of multicollinearity, the A-optimal design from Table 7 clearly outperforms the two benchmark designs.

Table 8 shows the variances of the parameter estimates from the three 12-run 4-factor designs in Table 7 . The variances of the factor-effect estimates for the A-optimal design are all smaller than those for the orthogonal array. For the A-optimal design, the variances of the factor effects' variances are either $1 / 8=0.125$ or $3 / 32 \approx 0.094$, while, for the orthogonal array, they all equal $13 / 96=0.135$. For two main-effect estimates and three interaction-effect estimates, the variances are smaller when using the A-optimal design than when using the D-optimal design (since $3 / 32 \approx 0.094,1 / 8=0.125$ and $15 / 128 \approx 0.117$ ). For two main-effect estimates and two interaction-effect estimates, the variances are equal to $1 / 8$ for the two designs, and, for one interaction-effect estimate, the D-optimal design produces the smallest variance $(15 / 128 \approx 0.117$ versus $1 / 8=0.125)$. The $\mathrm{D}$-optimal design leads to the least precise intercept estimate of the three designs under comparison. 


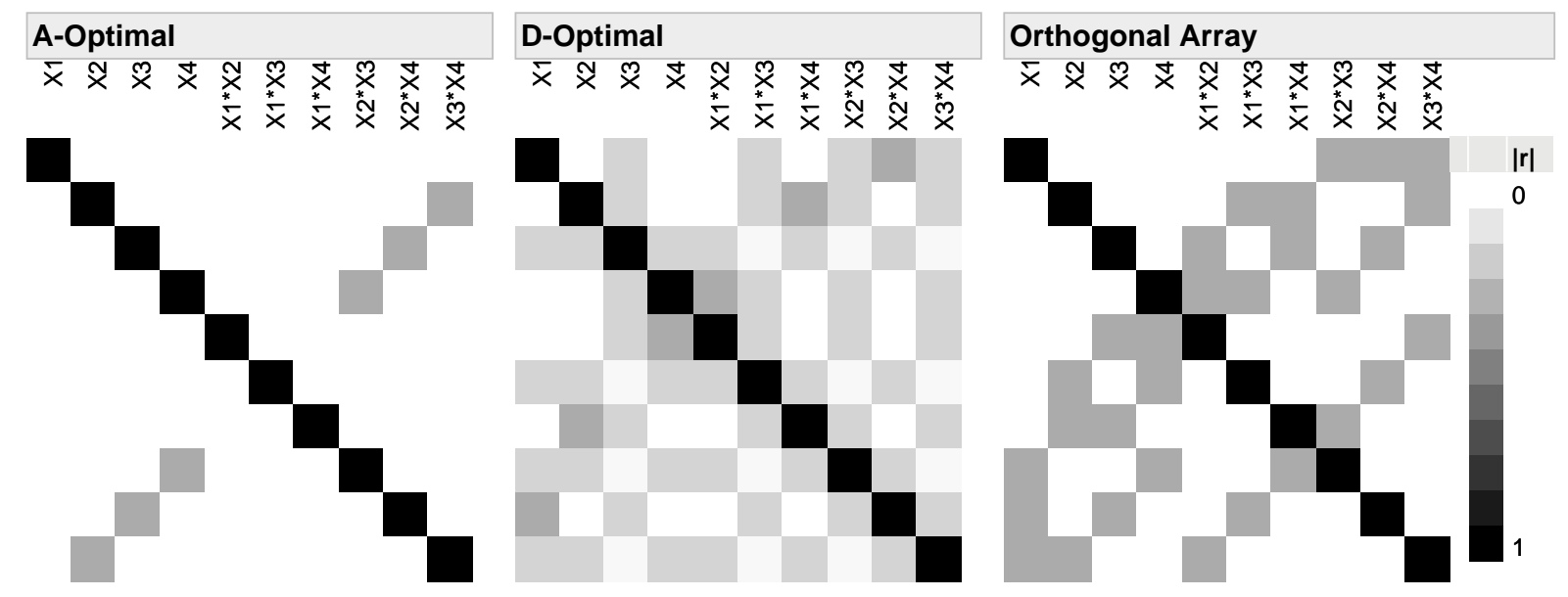

Figure 4: Color maps of the correlations between the main-effects and two-factor-interaction columns of the three designs in Table 7.

As shown in Table 9, the A-optimal design from Table 7 outperforms the D-optimal design and the orthogonal array in terms of A-efficiency, by $14.6 \%$ and $25.5 \%$, respectively. The I-efficiency and the G-efficiency are also in favor of the A-optimal design. The Aoptimal design outperforms both benchmark designs by $87.5 \%$ in terms of G-efficiency. Finally, the difference in D-efficiency between the three designs is not as large as that in A-, I- and G-efficiency. The A-optimal design is $2.6 \%$ worse than the D-optimal design in terms of D-efficiency, and $2.7 \%$ better than the orthogonal array. In this example, the A-optimal design is not D-optimal.

\subsubsection{Example 5: 28 runs and 6 factors}

In our last example, we compare an A-optimal 28-run 6-factor design with a D-optimal design and with an orthogonal array. The A- and D-optimal designs we obtained from JMP are shown in Table 10, as well as our benchmark orthogonal array. The benchmark orthogonal array is the design labeled OA \#17825 in Jones et al. (2016). These authors identified that array as the most A- and D-efficient 28-run 6-factor two-level orthogonal array for estimating the main-effects-plus-interactions model.

The color maps visualizing the absolute correlations between the main-effects and twofactor-interaction columns of the model matrices for the three designs under comparison 
Table 8: Variances of the parameter estimates for the three designs in Table 7.

\begin{tabular}{lccc}
\hline Term & A-Optimal & D-Optimal & Orth. Array \\
\hline Intercept & $1 / 12$ & $3 / 32$ & $1 / 12$ \\
$x_{1}$ & $1 / 8$ & $1 / 8$ & $13 / 96$ \\
$x_{2}$ & $3 / 32$ & $1 / 8$ & $13 / 96$ \\
$x_{3}$ & $3 / 32$ & $15 / 128$ & $13 / 96$ \\
$x_{4}$ & $1 / 8$ & $1 / 8$ & $13 / 96$ \\
$x_{1} x_{2}$ & $1 / 8$ & $1 / 8$ & $13 / 96$ \\
$x_{1} x_{3}$ & $1 / 8$ & $15 / 128$ & $13 / 96$ \\
$x_{1} x_{4}$ & $1 / 8$ & $1 / 8$ & $13 / 96$ \\
$x_{2} x_{3}$ & $3 / 32$ & $15 / 128$ & $13 / 96$ \\
$x_{2} x_{4}$ & $3 / 32$ & $1 / 8$ & $13 / 96$ \\
$x_{3} x_{4}$ & $3 / 32$ & $15 / 128$ & $13 / 96$ \\
\hline
\end{tabular}

are shown in Figure 5. The A-optimal design again has the largest number of pairs of orthogonal columns. It has 186 such columns, compared to 40 for the D-optimal design and 105 for the orthogonal array. The A-optimal design produces three pairs of columns with an absolute correlation of $1 / 3$, while the two other designs have maximum absolute correlations of 0.149 and $1 / 7 \approx 0.143$, respectively. The orthogonal array produces 105 pairs of columns with absolute correlations of $1 / 7$, while the D-optimal design produces 100 pairs with absolute correlations of $1 / 14=0.071$. The average absolute correlation is

Table 9: Efficiencies of the A-optimal design relative to the D-optimal design and the orthogonal array in Table 7.

\begin{tabular}{ccc}
\hline Criterion & Relative Efficiency D & Relative Efficiency OA \\
\hline D & 0.9742 & 1.0265 \\
G & 1.8750 & 1.8750 \\
A & 1.1455 & 1.2545 \\
I & 1.1607 & 1.2143 \\
\hline
\end{tabular}


Table 10: A-optimal and D-optimal 28-run, 6-factor designs for the main-effects-plusinteractions model, and orthogonal array \#17825 from Jones et al. (2016).

\begin{tabular}{|c|c|c|c|c|c|c|c|c|c|c|c|c|c|c|c|c|c|}
\hline$x_{1}$ & $x_{2}$ & $x_{3}$ & $x_{4}$ & $x_{5}$ & $x_{6}$ & $x_{1}$ & $x_{2}$ & $x_{3}$ & $x_{4}$ & $x_{5}$ & $x_{6}$ & $x_{1}$ & $x_{2}$ & $x_{3}$ & $x_{4}$ & $x_{5}$ & $x_{6}$ \\
\hline 1 & -1 & 1 & 1 & 1 & 1 & 1 & 1 & -1 & 1 & -1 & 1 & -1 & -1 & -1 & -1 & -1 & -1 \\
\hline 1 & 1 & 1 & 1 & -1 & 1 & -1 & 1 & 1 & -1 & -1 & -1 & -1 & -1 & -1 & -1 & -1 & 1 \\
\hline-1 & -1 & -1 & 1 & -1 & 1 & -1 & -1 & 1 & 1 & 1 & -1 & -1 & -1 & -1 & -1 & 1 & -1 \\
\hline 1 & 1 & 1 & 1 & 1 & -1 & -1 & 1 & -1 & -1 & 1 & -1 & -1 & -1 & -1 & 1 & -1 & 1 \\
\hline 1 & 1 & -1 & 1 & 1 & -1 & -1 & -1 & -1 & -1 & -1 & 1 & -1 & -1 & 1 & -1 & 1 & -1 \\
\hline-1 & -1 & 1 & 1 & -1 & 1 & -1 & 1 & 1 & -1 & 1 & 1 & -1 & -1 & 1 & 1 & -1 & -1 \\
\hline-1 & 1 & 1 & -1 & -1 & 1 & 1 & 1 & 1 & -1 & 1 & -1 & -1 & -1 & 1 & 1 & 1 & 1 \\
\hline 1 & -1 & 0 & -1 & -1 & 1 & 1 & -1 & -1 & -1 & 1 & 1 & -1 & 1 & -1 & -1 & 1 & 1 \\
\hline-1 & 1 & -1 & -1 & -1 & 1 & -1 & -1 & 1 & 1 & -1 & 1 & -1 & 1 & -1 & 1 & -1 & 1 \\
\hline-1 & -1 & 1 & -1 & 1 & 1 & -1 & -1 & -1 & -1 & 1 & -1 & -1 & 1 & -1 & 1 & 1 & -1 \\
\hline-1 & -1 & 1 & 1 & 1 & -1 & -1 & 1 & -1 & 1 & -1 & 1 & -1 & $x$ & 1 & -1 & -1 & -1 \\
\hline 1 & -1 & -1 & 1 & -1 & -1 & 1 & 1 & 1 & -1 & -1 & 1 & -1 & 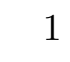 & 1 & -1 & -1 & 1 \\
\hline-1 & 1 & -1 & 1 & 1 & 1 & 1 & 1 & 1 & 1 & -1 & -1 & -1 & 1 & 1 & 1 & 1 & -1 \\
\hline 1 & 1 & 0 & -1 & 1 & 1 & 1 & 1 & -1 & -1 & -1 & -1 & -1 & 1 & 1 & 1 & 1 & 1 \\
\hline 1 & 1 & 1 & -1 & -1 & -1 & -1 & -1 & 1 & -1 & -1 & -1 & 1 & -1 & -1 & -1 & 1 & 1 \\
\hline 1 & -1 & -1 & -1 & 1 & 1 & -1 & 1 & 1 & 1 & 1 & -1 & 1 & -1 & 1 & 1 & -1 & -1 \\
\hline-1 & -1 & 0 & -1 & -1 & 1 & 1 & -1 & -1 & -1 & -1 & -1 & 1 & -1 & -1 & 1 & 1 & -1 \\
\hline-1 & -1 & -1 & -1 & 1 & 1 & 1 & -1 & 1 & -1 & -1 & 1 & 1 & -1 & 1 & -1 & -1 & 1 \\
\hline 1 & 1 & -1 & -1 & -1 & -1 & 1 & 1 & -1 & 1 & 1 & -1 & 1 & -1 & 1 & -1 & 1 & -1 \\
\hline-1 & 1 & 1 & 1 & 1 & 1 & 1 & 1 & -1 & -1 & 1 & 1 & 1 & -1 & 1 & 1 & -1 & 1 \\
\hline-1 & 1 & -1 & 1 & -1 & -1 & 1 & 1 & 1 & 1 & 1 & 1 & 1 & -1 & 1 & 1 & 1 & 1 \\
\hline-1 & 1 & 1 & 1 & -1 & -1 & 1 & -1 & -1 & 1 & 1 & -1 & 1 & 1 & -1 & -1 & -1 & 1 \\
\hline 1 & -1 & 1 & 1 & -1 & -1 & 1 & -1 & 1 & 1 & -1 & -1 & 1 & 1 & -1 & -1 & 1 & -1 \\
\hline 1 & -1 & -1 & 1 & 1 & 1 & 1 & -1 & -1 & 1 & -1 & 1 & 1 & 1 & -1 & 1 & -1 & -1 \\
\hline 1 & 1 & -1 & 1 & -1 & 1 & -1 & -1 & 1 & -1 & 1 & 1 & 1 & 1 & -1 & 1 & 1 & 1 \\
\hline 1 & -1 & 1 & -1 & 1 & -1 & 1 & -1 & 1 & 1 & 1 & 1 & 1 & 1 & 1 & -1 & -1 & -1 \\
\hline-1 & 1 & 0 & -1 & 1 & -1 & -1 & -1 & -1 & 1 & -1 & -1 & 1 & 1 & 1 & -1 & 1 & 1 \\
\hline-1 & -1 & -1 & 1 & 1 & -1 & -1 & -1 & -1 & 1 & 1 & 1 & 1 & 1 & 1 & 1 & -1 & -1 \\
\hline
\end{tabular}



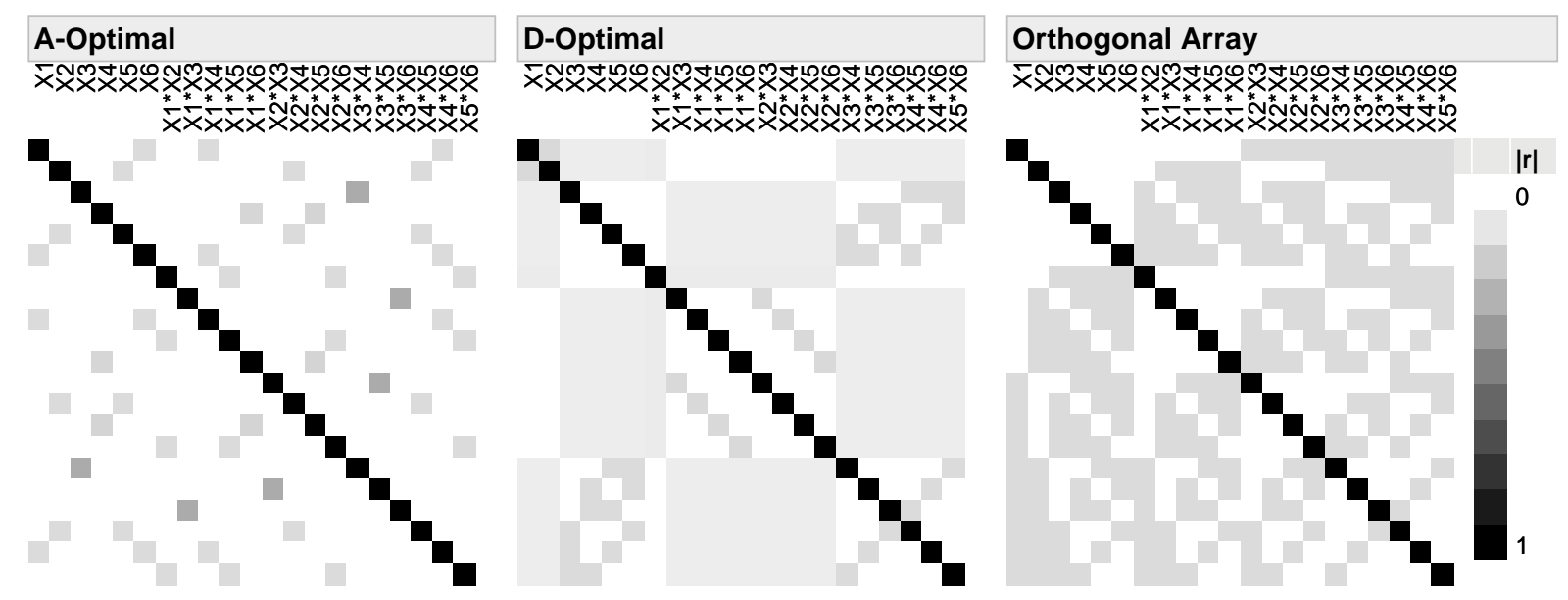

Figure 5: Color maps of the correlations between the main-effects and two-factor-interaction columns of the 28-run, 6-factor designs in Table 10.

0.019, 0.053 and 0.071 for the A-optimal design, the D-optimal design and the orthogonal array, respectively, indicating that the A-optimal design involves the smallest degree of multicollinearity.

When comparing the variances of the estimates, the A-optimal design outperforms the orthogonal array for each of the model parameters other than the intercept. The difference in estimation efficiency goes up to $20.7 \%$, in favor of the A-optimal design. Comparing the A-optimal design with the D-optimal design, 15 of the 22 model parameters are estimated more precisely using the A-optimal design. The remaining six parameters are estimated more precisely using the D-optimal design. The most precise estimate of the intercept is produced by the orthogonal array. Table 11 lists the estimates' variances for the three designs under comparison here. On average, the variances equal 0.0412, 0.0421 and 0.0466 for the A-optimal design, the D-optimal design and the orthogonal array, respectively. The relative A-efficiency of the D-optimal design and the orthogonal array are therefore $97.9 \%$ and $88.4 \%$, respectively.

In this example, the A-optimal design is only a few percent better than the D-optimal design in terms of A-efficiency. A comparison of the two designs in terms of I-efficiency yields the same conclusion. The A-optimal design is about $2 \%$ worse than the D-optimal design in terms of D-efficiency. In terms of G-efficiency, the A-optimal design is $23.8 \%$ 
Table 11: Variances of the parameter estimates for the three designs in Table 10.

\begin{tabular}{|c|c|c|c|}
\hline Term & A-Optimal & D-Optimal & Orth. Array \\
\hline Intercept & 0.0391 & 0.0399 & 0.0357 \\
\hline$x_{1}$ & 0.0391 & 0.0414 & 0.0471 \\
\hline$x_{2}$ & 0.0391 & 0.0414 & 0.0471 \\
\hline$x_{3}$ & 0.0469 & 0.0432 & 0.0471 \\
\hline$x_{4}$ & 0.0391 & 0.0432 & 0.0471 \\
\hline$x_{5}$ & 0.0391 & 0.0432 & 0.0471 \\
\hline$x_{6}$ & 0.0391 & 0.0432 & 0.0471 \\
\hline$x_{1} x_{2}$ & 0.0391 & 0.0399 & 0.0471 \\
\hline$x_{1} x_{3}$ & 0.0469 & 0.0414 & 0.0471 \\
\hline$x_{1} x_{4}$ & 0.0391 & 0.0414 & 0.0471 \\
\hline$x_{1} x_{5}$ & 0.0391 & 0.0414 & 0.0471 \\
\hline$x_{1} x_{6}$ & 0.0391 & 0.0414 & 0.0471 \\
\hline$x_{2} x_{3}$ & 0.0469 & 0.0414 & 0.0471 \\
\hline$x_{2} x_{4}$ & 0.0391 & 0.0414 & 0.0471 \\
\hline$x_{2} x_{5}$ & 0.0391 & 0.0414 & 0.0471 \\
\hline$x_{2} x_{6}$ & 0.0391 & 0.0414 & 0.0471 \\
\hline$x_{3} x_{4}$ & 0.0469 & 0.0432 & 0.0471 \\
\hline$x_{3} x_{5}$ & 0.0469 & 0.0432 & 0.0471 \\
\hline$x_{3} x_{6}$ & 0.0469 & 0.0432 & 0.0471 \\
\hline$x_{4} x_{5}$ & 0.0391 & 0.0432 & 0.0471 \\
\hline$x_{4} x_{6}$ & 0.0391 & 0.0432 & 0.0471 \\
\hline$x_{5} x_{6}$ & 0.0391 & 0.0432 & 0.0471 \\
\hline
\end{tabular}


Table 12: Efficiencies of the A-optimal design relative to the D-optimal design and the orthogonal array in Table 10.

\begin{tabular}{ccc} 
Criterion & Relative Efficiency D & Relative Efficiency OA \\
\hline D & 0.982 & 1.035 \\
G & 1.238 & 1.111 \\
A & 1.021 & 1.132 \\
I & 1.031 & 1.102 \\
\hline
\end{tabular}

better than the D-optimal design. When compared to the orthogonal array, the A-optimal design is $11.1 \%$ more G-efficient, $13.2 \%$ better in terms of A-efficiency, $10.2 \%$ better in terms of I-efficiency and $3.5 \%$ better in terms of D-efficiency. Note that, here too, the A-optimal design is not D-optimal.

\section{Discussion}

In this article, we compared A- and D-optimal screening designs. We first focused on the main-effects model, and provided an overview of existing results concerning A-optimal designs for that model. We explained that, unless the number of experimental runs is three more than a multiple of four, A- and D-optimal designs coincide for the main-effects model. Next, we turned our attention to the main-effects-plus-interactions model. For that model, A- and D-optimal designs can differ regardless of the run size. In each of the cases where we found differences between A- and D-optimal designs, the A-optimal designs were preferable to the D-optimal designs. In summary, A-optimal screening designs are, according to our computational results, at least as good as D-optimal designs. Therefore, in our opinion, there is no reason to stick to the traditional D-optimality criterion for designing screening experiments.

We described several cases where the traditional D-optimal two-level designs and Aoptimal designs for the main-effects model are different. In each of these cases, adding one extra run would allow for an orthogonal main-effects plan, which would be both A- and D-optimal for the new number of runs. If adding an extra run were feasible, we would 
certainly approve that choice. However, there are three scenarios in which this action may not be possible. First, in the case where each run is prohibitively expensive, management may be unwilling to add budget to pay for the extra run. Second, access to the system under study may be limited due to the need to meet production deadlines, resulting in an upper bound on the number of runs. Finally, it may be the case that a limited number of experimental units are available at the time of implementation. Thus, cost or logistical concerns may override all others.

We also supplied two illustrative examples of designs for the main-effects-plus-interactions model. The final example with six factors and 28 runs shows the clear advantages an Aoptimal design can have over both a D-optimal design and an orthogonal array that is best in some sense. In this example, adding four zero settings for one of the factors provides a compelling effect on the number of zero pairwise correlations between the columns of the model matrix. This can simplify the eventual task of model selection.

The beneficial effect of the A-optimality criterion on the pairwise correlations between the columns of the model matrix was a recurring theme in all of our examples in the paper. In most examples, A-optimal designs also performed better in terms of I- and G-efficiency than D-optimal designs, while being highly D-efficient or even D-optimal themselves. The gain in G-efficiency was especially striking in several of the examples. We were able to confirm these observations through a systematic study of A- and D-optimal designs for different numbers of runs and factors.

A technical explanation of why A-optimal designs produce fewer nonzero pairwise correlations than D-optimal designs is as follows. A D-optimal design maximizes the determinant of the information matrix, which is equivalent to minimizing the determinant of the ordinary least squares estimator's variance-covariance matrix. It therefore provides the smallest possible product of the variances among all possible designs with respect to a rotation of the parameter axes yielding a set of mutually orthogonal vectors. In cases where all the columns of the model matrix are mutually orthogonal, no rotation is necessary and the product of the variances is $1 / N^{p}$, which is the minimum possible value when all entries in the model matrix are either 1 or -1 . When the columns of the model matrix of a D-optimal design are not orthogonal, the rotated vectors may not be close to the parameter axes and 
may have no meaning in an engineering sense. In such a case, the vector of variances of the parameter estimates is more meaningful and a useful scalar summary is their average. What an A-optimal design does is minimize the average variance of the parameter estimates over all possible designs for the same model and number of runs. In so doing, an A-optimal design minimizes those variances that are meaningful in an engineering sense, which results in fewer nonzero correlations between pairs of columns in the model matrix.

The excellent performance of A-optimal designs in terms of the other optimality criteria is unexpected, since it is D-optimal designs that are generally credited for performing well in terms of other criteria. It turns out that, for screening experiments at least, A-optimal designs do better in this respect. Another surprising aspect of our results is that A-optimal designs often outperform D-optimal ones in terms of G-efficiency, by a large margin. Due to the general equivalence theorem (Kiefer and Wolfowitz; 1960), D- and G-optimal designs are equivalent in the event a large number of runs are available. It is therefore tempting to expect that D-optimal designs will perform well in terms of G-efficiency, even when the number of runs is not large. However, it now turns out that A-optimal design often do a better job at lowering the maximum variance of prediction than D-optimal designs.

Finally, we have two concluding remarks concerning the D-optimality criterion. First, for several of the cases we explored in detail, we observed that many distinct D-optimal designs exist and that these designs perform substantially differently in terms of other criteria. This suggests that the D-optimality criterion is not capable of distinguishing between screening designs that are clearly not equally good in an overall sense. The A-optimality criterion seems to do a better job in this respect. Second, our research revealed that Doptimal designs for main-effects models and main-effects-plus-interactions models are not necessarily two-level designs. On multiple occasions, we encountered designs involving three levels that were D-optimal.

\section{Acknowledgement}

The authors are grateful for the helpful comments and input from Caleb King. 


\section{References}

Atkinson, A. C. and Donev, A. N. (1989). The construction of exact D-optimum experimental designs with application to blocking response surface designs, Biometrika 76: 515-526.

Atkinson, A. C. and Donev, A. N. (1992). Optimum Experimental Designs, Oxford U.K.: Clarendon Press.

Box, M. J. and Draper, N. R. (1971). Factorial designs, the $\left|X^{\prime} X\right|$ criterion, and some related matters, Technometrics 13: 731-742. Correction, 14, 511 (1972); 15, 430 (1973).

Cheng, C.-S. (1980). Optimality of some weighing and $2^{n}$ fractional factorial designs, Annals of Statistics 8(2): 436-446.

Cook, R. D. and Nachtsheim, C. J. (1980). A comparison of algorithms for constructing exact D-optimal designs, Technometrics 22: 315-324.

Cook, R. D. and Nachtsheim, C. J. (1989). Computer-aided blocking of factorial and responsesurface designs, Technometrics 31: 339-346.

Ehlich, H. and Zeller, K. (1962). Binäre matrizen, Zeitschrift für Angewandte Mathematik und Mechanik 42: T20-T21.

Galil, Z. and Kiefer, J. (1980). D-optimum weighing designs, The Annals of Statistics 8(6): 12931306.

Galil, Z. and Kiefer, J. (1982). Construction methods for D-optimum weighing designs when $n \equiv 3(\bmod 4)$, The Annals of Statistics 10(2): 502-510.

Goos, P. (2002). The Optimal Design of Blocked and Split-plot Experiments, New York: Springer.

Goos, P. and Jones, B. (2011). Design of Experiments: A Case-Study Approach, New York: Wiley.

Jacroux, M., Wong, C. and Masaro, J. (1983). On the optimality of chemical balance weighing designs, Journal of Statistical Planning and Inference 8(2): 231-240.

Jones, B., Schoen, E. D. and Montgomery, D. C. (2016). A comparison of two-level designs to estimate all main effects and two-factor interactions, Quality Engineering 28: 369-380. 
Kiefer, J. and Wolfowitz, J. (1960). The equivalence of two extremum problems, Canadian Journal of Mathematics 12: 363-366.

Masaro, J. C. (1983). Optimality of chemical balance weighing designs, Ph.D. dissertation, University of Windsor.

Mitchell, T. J. (1974). An algorithm for the construction of D-optimal experimental designs, Technometrics 16: 203-210.

Sathe, Y. S. and Shenoy, R. G. (1989). A-optimal weighing designs when $n \equiv 3(\bmod 4)$, Annals of Statistics 17: 1906-1915.

Schoen, E. D., Vo-Thanh, N. and Goos, P. (2017). Two-level orthogonal screening designs with 24, 28, 32, and 36 runs, Journal of the American Statistical Association 112(519): 1354-1369.

Wong, C. S. and Masaro, J. C. (1984). A-optimal design matrices $x=\left(x_{i j}\right)_{N \times n}$ with $x_{i j}=-1,0,1$, Linear and Multilinear Algebra 15(1): 23-46. 